\title{
Understanding Situated Social Interactions in Public Places
}

\author{
Jeni Paay ${ }^{1}$ and Jesper Kjeldskov ${ }^{2}$ \\ ${ }^{1}$ Department of Information Systems, The University of Melbourne, \\ Victoria, 3010, Australia \\ jpaay@unimelb.edu.au \\ 2 Department of Computer Science, Aalborg University, \\ 9220 Aalborg, Denmark \\ jesper@cs.aau.dk
}

\begin{abstract}
Designing context-aware mobile information systems for supporting sociality requires a solid understanding of the users' context, situated interactions, and the interplay between the two. Currently such understanding is lacking in the field of HCI research and is sought after by several authors. Addressing this gap we conducted a field study of small groups socialising in a public place. Based on a grounded analysis of our findings we present a conceptual framework of situated social interactions in public. Finally, we illustrate how this framework informed design of a mobile context-aware prototype.
\end{abstract}

\section{Introduction}

Mobile computer technologies are increasingly being appropriated and used to support people's social life outside the work domain. Mobile phones, and especially SMS (Short Messaging Service), have changed the way people communicate with each other, interact in the physical world and coordinate their social activities [10] [18]. Smart Phones and Personal Digital Assistants (PDAs) connected to the Internet bring web services to the mobile user and extend the potentials of SMS through Internetchat capabilities and facilities for video-based communication. By adding advanced positioning technology and short range network capabilities (such as Bluetooth) mobile services are beginning to appear which adapt their content to the user's physical and social context. SMS messages are being sent to customers in shopping centres and airports on the basis of their position. Mobile dating services exist which alert the user when they are in the proximity of a potential partner who matches their own pattern of attributes [6]. In the more experimental domain mobile guide systems provide information about the location of friends in vicinity [9], take into consideration the user's social context [4] and enable people to attach small messages for each other (virtual graffiti) to physical locations for other people to find when they enter that specific place [16].

When developing such systems designers are faced with a huge challenge. How do we take into consideration the user's physical and social context in our interaction design in a way that makes sense and is useful to the user? In order to answer this question we need to understand better the user's physical and social context, their 
situated social interactions [14], the role of human activity within the built environment [5] and the interplay between context and user actions [8]. We need to study how physical and social affordances of a place influence the situated interactions that occur there and we must understand the relationship between interactions in the built environment and the social roles and rules of the people who inhabit that space [1]. Also, we need to understand the social processes that surround our everyday interactions with others [22].

This paper addresses this challenge by providing an understanding of peoples social interaction in a public place derived through grounded theory from a field study of small groups of people socialising in public. It also illustrates how this understanding informed the interaction design of a prototype system. The paper is structured in the following way. Section 2 briefly introduces the concept of situated interactions and the typology of situated interactions proposed by McCullough [12]. In section 3 we present our field study of socialising in public, describing the details of our empirical method and data analysis. In section 4 we present the findings from our study in the form of a conceptual framework of situated interactions in public places. Illustrating the use of this model for informing interaction design, section 5 describes the basic design ideas of an implemented prototype system for Federation Square which adapts to the user's physical and social context. Section 6 concludes on this study and outlines our current research directions.

\section{Situated Interactions}

Recent research approaches into context-aware computing have focused on recurrent patterns of everyday life and the relation between interactions of people and technology and the social settings in which these interactions take place [7]. Dourish regards context as a central concept in social analyses of interaction and says that social and cultural factors affect how the user makes decisions about actions and interprets a system. It seems important therefore to understand the social context of use for a mobile system to be able to predict how users will perceive it. This approach to defining context is further explored by Dourish [8] where he regards the operational situation of context-aware technology as "varied" with context being particular to each occasion of activity or action, requiring mobile and ubiquitous systems to be more responsive to the different social settings in which they might be used. Studying people's "everyday action" is one way to be able to provide designers with a sense of the meaning associated with user activities, an understanding of people's experience of place and knowledge about what they actually do in a particular situation.

One of the challenges of modelling social situated interaction is finding a method of representation to capture the situational and social aspects of a space that influence people's ability to achieve their intended activities within that built environment. In response to this McCullough [12] offers the idea of using typology (the study of recurrent forms) as a design philosophy and provides types of everyday situations as a way of abstracting context for context-aware computer applications. In his recent book McCullough [14] elaborates on this set of situational types and talks about computing as consisting of situations, rather than objects, and context as not being just the setting in which computing is embedded, but rather the user's engagement with that setting affecting the interactions that occur there. He characterizes a new era in infor- 
mation technology that focuses on experience and the need to understand how people play out situations. Social situations are seen as providing design precedents and problems from which to build types that can be used in creating a new form of context-centred design. Better modelling of people in contexts is the best way towards more human-centred design of mobile and pervasive computing systems.

In providing a preliminary list of everyday situations that may be transformed by technologies McCullough [12] presents a rudimentary typology of 30 different situations, a typology of "life's habitual places", grouped to reflect the usual categories of place: at work, at home, on the town and on the road. However, the use of this typology for informing interaction design for sociality is yet to be explored. As a starting point, and with the aim of understanding sociality in public places, we have focused on the situated interactions associated with being "on the town". These can be summarised briefly as: 1) eating, drinking, talking (places for socializing); 2) gathering (places to meet); 3) cruising (places for seeing and being seen); 4) belonging (places for insiders); 5) shopping (places for recreational retailing); 6) sporting (places for embodied play); 7) attending (places for cultural productions); and 8) commemorating (places for ritual).

\section{Field Study: Socialising at Federation Square}

Exploring the categories of McCullough's typology of "on the town" everyday situations listed above, we conducted a field study of social interaction at Federation Square, Melbourne, Australia. Federation Square is a new civic structure covering an entire city block, providing the people of Melbourne with a mixture of digital and architectural elements that provides a variety of activities to visitors including restaurants, cafes, bars, a museum, galleries, cinemas, retail shops and several public forums. The intention for the space was to incorporate digital technologies into the building fabric creating a combination of virtual information space and physical building space for people to experience.

\subsection{Participants, Procedure and Data Collection}

Our field study consisted of a series of contextual interviews [2] and ethnographic field observations [3] on location at Federation Square. Three different established social groups participated in the study. Each group consisted of three young urban people, mixed gender, between the ages of 20 and 35, who had a shared history of socializing at Federation Square together. Prior to the field visits each group received a 10 minute introduction to the study followed by a 20 minute interview about their socializing experiences and preferences, about places familiar to the group. One of the members of the group was then taken to Federation Square and asked to contact the other members of the group and arrange to meet up with them as they would usually do when out on the town. When the group had met at Federation Square they were not given any further assignments but were asked to simply undertake the same activities that they would usually do as a group when socialising at Federation Square. Furthermore, the participants were asked to "think aloud" as they moved around the space and respond to an interviewer interjecting with questions on points of clarification about things that had been said or decisions and interactions that were not so explicit, as they involved 
themselves in different interactions and activities (see figure 1). The contextual interviews and observations lasted approximately three hours for each visit.

One researcher managed and conducted the contextual interviews while the other recorded the interaction between the group and the interviewer on digital video and audio. The outcome from the field visits amounted to: 8 hours of digital video documenting all questions, responses, initiation of activities and movement of the group around the square; notes of field observations; and diary reflections about each visit recorded immediately after it.

The first group participated in two visits to Federation Square. However, during the repeat visit their enthusiasm for participation in the study observably waned and the observers learned only very little new about their social interactions relative to the time commitment required from the participants. On the basis of this lesson, the second and third groups were only asked to do a single visit each.

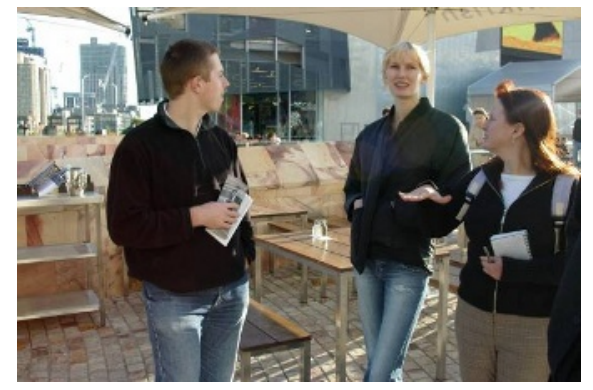

Fig. 1. Contextual Interview

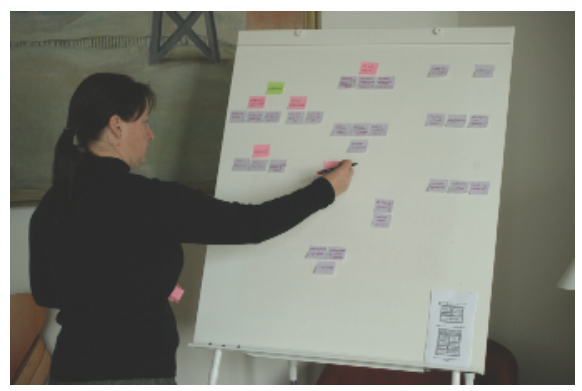

Fig. 2. Affinity Diagramming

\subsection{Transcriptions and Data Analysis}

Shortly after the field visits all video recordings were reviewed, transcribed and analysed. The review and transcription process consisted of three steps. Firstly, each recording was viewed in its entirety during which hand written notes were made noting the place of situated interactions and any interesting events observed. These notes were combined with field notes made during the visit. Secondly, an electronic log-file was created noting the time stamp on the tape where each situated interaction event started. Thirdly, all spoken interactions between interviewer and participants including gestures and actions (such as pointing and the group forming a closed circle) were transcribed. The full transcription only covered conversations related to the participants' situated interactions. The transcription files contained the following columns: 1) time stamp (derived from the video recording); 2) situated interaction (listing situated interaction type); 3) transcript (the spoken words and actions relating to that situated interaction); and 4) margin note (notes made during transcription about initial analytical thoughts or identifying interesting trends in the data). During the later analysis phase the following columns were added: 5) codes (created through open coding); and 6) themes (higher-level categories and themes).

The analysis of the transcript involved open coding adapted from the grounded theory analysis method [15] [21] and affinity diagramming [2] (see figure 2). The 
open coding of the first field visit began before the field visits with the other two groups were completed. Identifying key words or events in the transcript and analyzing the underlying phenomenon being represented created the codes. To ensure consistent use of codes, each code was supplemented with a brief explanation. By reviewing the codes higher-level categories were drawn out, describing situated interactions and actions, with properties and dimensions of the category carrying the detail of the phenomenon. On the basis of this, high-level themes were then extracted from the data using axial coding and looking at the relationships across the data and between occurrences of composite descriptive categories.

The transcript from the first group produced 73 novel themes. The second group produced 34 novel themes and the third group did not contribute with any significantly new themes. One researcher did the transcription, coding and themeing. Following the coding of the data the themes were transferred to individual pieces of paper for the process of affinity diagramming. Another researcher carried out axial coding and independently analyzed and grouped the themes to produce a set of higherlevel concepts. Both authors then worked cooperatively on this grouping, debating and refining each cluster of themes until consensus was reached. Affinity diagramming was used to draw successively higher levels of abstraction from the data by grouping and sorting the themes until a small set of high-level concepts, representing the essence of the data and encompassing all lower level themes, was extracted.

This affinity diagramming process resulted in a conceptual framework containing four levels of grouped themes abstracted above the themes identified in the transcripts. The outcome of the analysis is described in section 4 below.

\section{Situated Social Interaction in Public Places}

The conceptual framework that emerged from the analysis of situated interactions at Federation Square is called SOPHIA (SOcial PHysical Interaction Analysis). SOPHIA encapsulates a formalized understanding of every day social interaction in the situation of a public place. At the same time SOPHIA also contains a rich understanding of the role of physical and social context in the form of a structured qualitative story about how people experience physical space and how they interact with each other while socializing in these spaces.

SOPHIA consists of seven high level themes grouped into three main aspects of social interaction in the physical setting of a public place: knowledge, context and motivation. The complete SOPHIA framework is presented in Table 1 and explained in detail below.

\subsection{SOPHIA: Knowledge}

People socially interacting in public places draw on their knowledge using their understanding of the world around them, that is, their knowledge-in-the-world. They use physical affordances to operate in the world recognizing places for entering or places for gathering or they use landmarks as focal points. People also operate in public places using a set of social affordances. They look to what other people are doing to find cues about what to do in a place. Following crowds or people queuing is a way for people to work out where they are supposed to go. 
Table 1. SOPHIA conceptual framework

\begin{tabular}{|c|c|c|c|}
\hline \multirow{11}{*}{ Knowledge } & \multirow{5}{*}{$\begin{array}{l}\text { knowledge-in- } \\
\text { the-world }\end{array}$} & \multirow{3}{*}{ physical affordances } & places to enter \\
\hline & & & places for gathering \\
\hline & & & landmarks as focal points \\
\hline & & \multirow[t]{2}{*}{ social affordances } & cues for what to do \\
\hline & & & cues for where to go \\
\hline & \multirow{6}{*}{ history } & \multirow[t]{2}{*}{ physical familiarity } & familiar paths \\
\hline & & & familiar places \\
\hline & & \multirow{4}{*}{ social experience } & past experience \\
\hline & & & shared experience \\
\hline & & & recommendations (experience of others) \\
\hline & & & preferences \\
\hline \multirow{11}{*}{ Context } & \multirow{4}{*}{ people } & \multirow{4}{*}{$\begin{array}{l}\text { us and them } \\
\text { (group and others) }\end{array}$} & interaction by maintaining group \\
\hline & & & interaction by proximity \\
\hline & & & interaction by watching \\
\hline & & & discomfort of waiting (waiting alone) \\
\hline & \multirow{3}{*}{ situation } & \multirow{3}{*}{ setting matters } & others (social) \\
\hline & & & environment (physical) \\
\hline & & & convenience \\
\hline & \multirow{4}{*}{ surroundings } & \multirow{4}{*}{$\begin{array}{l}\text { indexing to sur- } \\
\text { roundings }\end{array}$} & index to shared knowledge \\
\hline & & & index to visible elements \\
\hline & & & index to events \\
\hline & & & index to physical objects \\
\hline \multirow{14}{*}{ Motivation } & \multirow{8}{*}{ reflection } & \multirow{4}{*}{$\begin{array}{l}\text { sizing up the } \\
\text { situation }\end{array}$} & getting an overview \\
\hline & & & pausing before committing \\
\hline & & & making sense of a place \\
\hline & & & making sense of what's happening \\
\hline & & \multirow{4}{*}{$\begin{array}{l}\text { seeking } \\
\text { information }\end{array}$} & different levels of information \\
\hline & & & media screens as decoration \\
\hline & & & what's new \\
\hline & & & uncertainty (lack of information) \\
\hline & \multirow{5}{*}{ extension } & \multirow{3}{*}{ directed movement } & transition through spaces \\
\hline & & & dynamics of a place \\
\hline & & & wayfinding \\
\hline & & \multirow[t]{2}{*}{ exploring } & exploration for the sake of it \\
\hline & & & \\
\hline & \multirow{2}{*}{ negotiation } & \multirow{2}{*}{ making decisions } & discussing suggestions \\
\hline & & & someone takes the lead \\
\hline
\end{tabular}

People rely on their past history with a place to determine the activities they participate in and the way that they operate in the place. Physical familiarity with a place means that they approach specific places using familiar paths, the way that they "usually come". They also tend to choose places to socialize based on places they are familiar with. They use social experience of places as a basis for selecting places to socialize in with friends using their personal past experience, their shared experience with this group of friends or recommendations from the friends they are with or others. Sometimes they have a preference for something that they know they like. When socializing with a particular group of friends, people often arrange to meet in a place where they "usually meet" together. 


\subsection{SOPHIA: Context}

When socializing the presence of people, including friends and strangers, influence the way that people behave and move through public places. A group of friends maintains their sense of "group" by the way that they physically locate themselves. As they move through a public place they walk abreast and when they stop to negotiate they gather in a circle. People like to be near others but not necessarily interacting directly with them, they prefer "socializing by proximity" to others without feeling the need to speak to them. People watch others, especially if they feel unobserved or they are waiting on their own. Waiting alone is an uncomfortable situation eased by appearing occupied or by being in a busy or familiar place.

Situation is important for socializing. The setting in which a particular activity takes place matters. The presence of others and the types of people influences the acceptability of a place. Physical comfort is also important, whether a place is sunny, sheltered, etc., influences the choice of location to socialize. A place may also be chosen for the convenience of its location to other activities. Personal preferences for types of activity or types of food also guides place selection.

Surrounds are part of people's context. They describe a location of a place unknown to a friend in terms of the places and activities of shared experience that they hold with that person. They also use visible elements and point to them or they might refer to generally known events or physical objects, including landmarks.

\subsection{SOPHIA: Motivation}

Reflection on current experience is part of socializing in a place. People try to size up the situation. They like to get an overview of what is happening in a place. Before entering a situation, they tend to stand on the outside to understand what is happening. They stand back and familiarize with situations and they pause before entering. People strive to make sense of things and places around them. This includes making sense of what is happening in a place by assessing the activities of others. Different levels of information are often required by different people for different activities. People want to know what is new. Media screens while ostensibly informative are often regarded as decoration. Lack of appropriate information in a place leads to uncertainty in understanding that place.

Extension of knowledge about a place motivates social activity. Movement by transition through spaces involves paths that have people and activities of interest along the way. These places are dynamic and paths can be altered by the presence of crowds and ad-hoc structures. In way finding people navigate by familiar paths and they look ahead for familiar objects or ask others. People spend time exploring, both physical space and shared knowledge. Exploration for the sake of it and wandering and browsing extend their knowledge of the space. Friends spend time negotiating as they move around a space deciding what to do and where to go next sometimes discussing suggestions as a group, other times someone takes the lead and others follow.

\section{Implications for Design}

Inquiring into the usefulness of the knowledge represented by SOPHIA for informing interaction design we conducted a two-day design workshop with the aim of develop- 
ing design ideas for a context-aware mobile information system supporting sociality when "out on the town". Following the design workshop several iterations of paperprototyping [20] turned the most promising design ideas into a high-fidelity paper prototype. Subsequently, the paper prototype was implemented as a functional web application running in Microsoft Pocket Internet Explorer on HP iPAQ h5550 using mySQL, PHP, pushlets and server-side applications for handling context-awareness and dynamic generation of maps and graphics. The system keeps track of the users' location, their current activity and friends within close proximity. It also keeps a history of the users' visits to places around the city. The technical details of the prototype are described in [12].

In the following sub-sections we describe four of the seven design ideas emerging from SOPHIA and illustrate some of the resulting paper prototype design:

- Indexing content to history and context;

- Indexing directions for way finding to familiar places;

- Representing current activities within close proximity;

- Supporting meeting up by communication about places, activities and time.

Each design idea was drawn directly from themes and categories in SOPHIA. These are italicized in the descriptions below.

Indexing Content to History and Context. Evidenced in the data by the way people make decisions about where to go, one of the most interesting findings was the importance of people's past experiences in terms of their existing knowledge, history of visits and social experience with places and their current social group. In the design workshop this finding was explored further using, among others, a Venn diagram to examine the relationship of experience between two people, A and B (see figure 3). Looking at the diagram from A's point of view, A has a past history which includes a number of familiar places. These places play an important role in A's mind, in comparison to non-familiar places, as places A knows very well, likes to go to, might recommend to others and knows the location of. A subset of A's history may be shared with B and represents shared experience which can be referred to through indexical relational descriptions such as "where we met last time". Finally, B may have a past history of familiar places that A has not been to. When A and B are socialising these places may be brought up by $\mathrm{B}$ as recommendations for new places for $\mathrm{A}$ to go (and visa versa).

On the basis of the overall design idea of indexing [11] content to the users' individual and shared histories, the paper prototype was designed to rank recommendations about places to go the basis of the user's knowledge and current context.

When a member of a social group (the user) selects a specific activity on the device (such as e.g. "having coffee", "having a drink", "eating" or "attending a cultural event") it presents a list of recommendations of places to go (see figure 4). Rather than sorting the list of places alphabetically, for example, it is sorted on the basis of the systems knowledge about user's familiar places (history), current physical and social setting (where the user is and who he is with) and the current environmental setting (e.g. weather conditions). Firstly, the list contains places where the user has been to before together with the people that he is currently socializing with (shared experiences). This is followed by places that all people in the current social group 
have been to before but have not been to together. Thirdly, the list contains places where the user has been before that none of the other people in the social group have visited (past experience). This is followed by places that the user has not been to but that other members of the current social group have (recommendations). Finally, the remaining places in the vicinity of the social group are displayed.

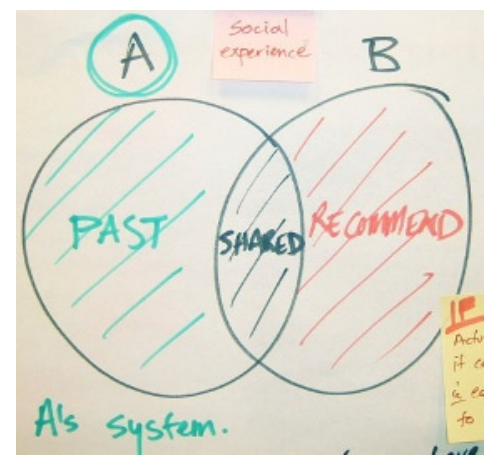

Fig. 3. Design workshop notes informed by SOPHIA: indexing to the users' individual and shared histories

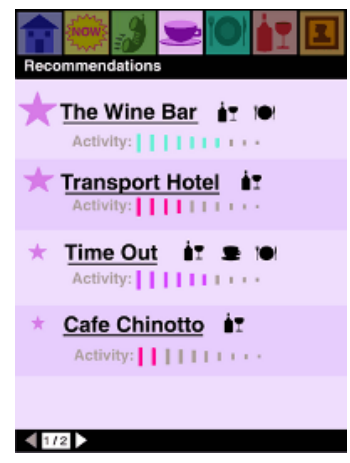

Fig. 4. Paper prototype: ranked list of recommendations

Within these sub-listings, places are ranked in consideration to the frequency of past visits, the proximity of places (convenience), the current activity at places and how well the weather situation of past visits to a place fits the current conditions. The highest scoring places are highlighted with a star next to the name. Furthermore, each place contains an "activity-meter" displaying the current busyness and primary activity. This ranking was informed by the findings that places and spaces are dynamic and that setting matters specifically in relation to environment, others and convenience. By providing this information the system supports the pervasive negotiation that happens when people are socializing together and exploring a space. It indexes to shared knowledge and gives the social group a chance to pause before committing to an activity or a place.

Indexing Directions for Way Finding to Familiar Places. In a somewhat similar fashion the data also evidences that people use their history and especially physical familiarity with a space as well as the knowledge-in-the world supported by physical affordances such as places to enter and landmarks to find their way around a space. Rather than requiring GPS-like instructions for way finding, people rely on simple indexing to their familiar places and prefer to follow their familiar paths from A to B even if this may not be the most time-efficient route. Furthermore, people often make use of their surrounds and index to visible elements and objects in their physical context. In the design workshop this finding was used to develop the idea of basing way finding on simple, indexical references to landmarks and familiar places with consideration to the user's history of familiar paths rather than efficiency (figure 5). This extends the mobile guide design presented in [16]. 


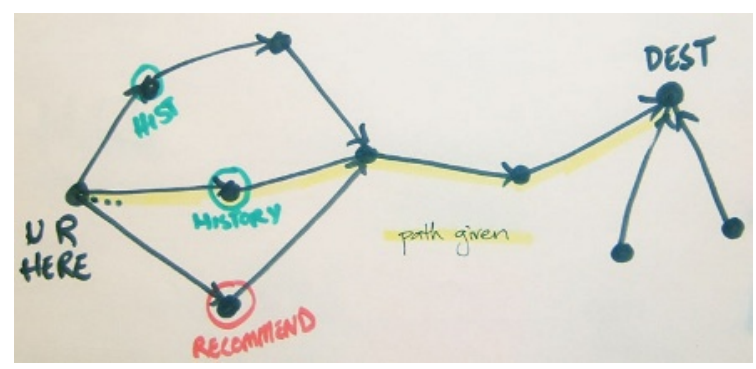

Fig. 5. Notes from design workshop informed by SOPHIA: indexing directions for way finding to familiar places and paths

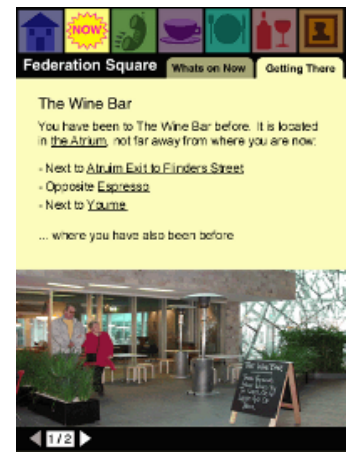

Fig. 6. Paper prototype: Indexical directions for way finding

Whenever the user accesses information about a place a "Getting There" pane becomes available. Clicking on this tab displays information about how to get to that place from the user's current location based on references to places where the user has been to before such as "Chocolate Buddha is located next to ACMI Cinemas opposite Arintji". If the place is not in the vicinity of anything known by the user the wayfinding descriptions indexes to places visible from both the destination and a familiar place or visible landmarks. If the place is not within close proximity to the user directions are divided into a series of sub pages guiding the user to the nearest familiar place or landmark. The way finding directions are combined with photographs of places, landmarks and transition points referred to in the text.

In this way, the system provides information that takes into consideration what people already know about the environment they are situated in and acknowledges their ability to make sense of an unfamiliar place on the basis of a few simple cues.

Representing Current Activities within Close Proximity. Another important observation made from our field study was the importance of knowing about the existence of other people in a space and what they are doing. Other people cohabiting a space play an important role in defining the social context by constituting an external frame of reference for the social group. The interaction between a social group and the other co-inhabitants of a space is complex. It involves maintaining a boundary between $u s$ and them while also allowing for a certain level of interaction between the group and others, either by proximity or by watching. Observing where other people are gathering and what they are doing there helps in getting an overview of a place, making sense of what is happening and sizing up the situation, which are a very important part of pausing before committing to enter a place. Observing, for example, other people gathering in an unfamiliar place may invoke exploration that in turn may result in an extension of a person's knowledge of that place. In the design workshop this finding was used to discuss and develop the idea of represent current activities of others within close proximity (figure 7). 

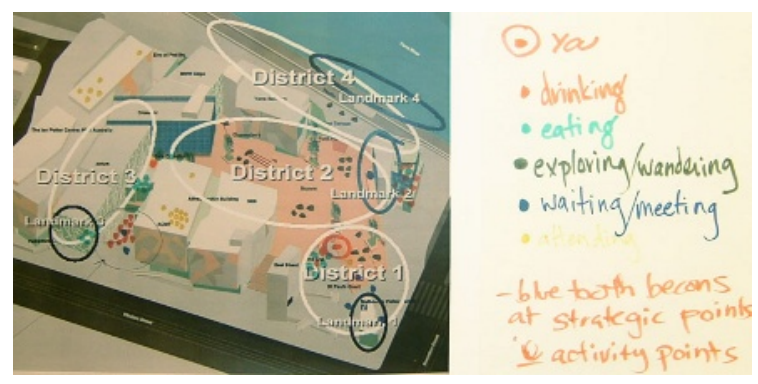

Fig. 7. Notes from design workshop informed by SOPHIA: representing current activities of others within close proximity

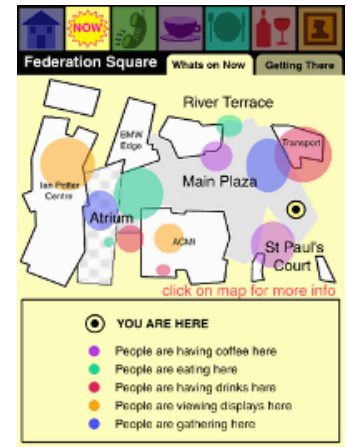

Fig. 8. Paper prototype: Dynamic activity map of places in vicinity

When the user clicks on the "NOW" icon on the main menu bar it displays a minimalistic map of the user's immediate surroundings. On this map superimposed, dynamically updated, circular coloured circles indicate the approximate location, current busyness and activities of places within proximity to the user. The radius of the circles represents the number of people at a place while the colour represents their primary current activity (e.g. "having coffee", "having a drink", "eating" or "attending a cultural event"). The colours of the circles match the system's general colour coding of activities and are explained through the legends at the bottom of the screen. The map also shows the location of the user. By clicking on the coloured circles the user can access more information about a place: detailed descriptions, photos, menus, programs, directions for way finding, etc.

In this way, the system supports making sense of a place through the social affordances provided by what others are doing and through access to different levels of information about places. It also accommodates people's desire for interaction between the group and others by proximity.

Supporting Meeting Up by Communication about Places, Activities and Time. A fourth finding from the data that had a major influence on the paper prototype is related to the activity of coordinating a rendezvous with your friends prior to socialising out on the town and the discomfort of waiting for the others to arrive at the agreed meeting point. When coordinating a rendezvous we found that people spend considerable effort on negotiating where to meet based on sometimes very complex and interrelated considerations about who they are meeting up with (people), what they want to do, where the others are coming from and how long they will be (convenience), where they have been together before (history), the weather conditions and what other people are doing around the considered meeting place (environmental and social setting). In extension of this, the discomfort of waiting recognizes peoples' need to look occupied and have something to do while waiting for their friends. When waiting alone people like to read newspapers, text their friends, talk on their mobile phones or watch the activities of others in order to look occupied. 


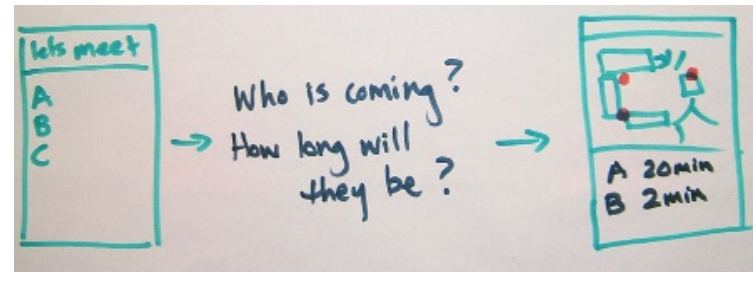

Fig. 9. Notes from design workshop informed by SOPHIA: supporting communication about people, places, activities and time

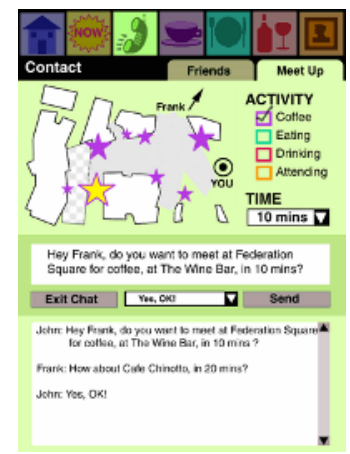

Fig. 10. Paper prototype: Context-aware chat $w$ autotext functions

When the user selects the "Contact" option on the top menu bar of the screen it displays a list of friends similar to the contacts list in e.g. MSN Messenger. The list is divided into three main parts: 1) friends who are online and within proximity of the user; 2) friends who are online but further away; and 3) friends who are offline. If two or more friends are currently together (within very close proximity of each other) they are displayed as a group. When the user selects a friend or group of friends the "Meet up" pane is activated and an Internet chat session is established (figure 10). At the receiving end this causes a brief ringing tone and a flashing telephone icon on the top of the screen. Apart from supporting free text input, the chat screen more importantly also supports automatic generation of small pieces of text with the purpose of supporting communication about people, places, activities and time. At the top of the screen a minimalistic map represents the user's immediate surroundings and the location of the participants in the chat (absolute if within the map area and relative if not). Next to this the user can choose between the activities supported by the places on the map. After selecting an activity, recommended places (generated on the basis of the user's history and context using the same algorithm for recommendations described earlier) are shown on the map by means of different sized coloured stars.

Having initiated a chat from John to Frank selecting, for example, the "coffee" checkbox, clicking on the star on the map representing "The Wine Bar" and choosing "10 min" from the time drop down menu will cause the following text "Hey Frank, do you want to meet at Federation Square for coffee at the wine bar in 10 minutes?" to be generated in the outgoing message window. Here the user can subsequently edit it. When an automatically generated text message is sent it causes the selected place, activity and time to be synchronized among the participants in the chat. The other participants in the chat can then modify the original suggestion by selecting another place, activity and time, causing a counter suggestion to be generated in the outgoing message window such as "No, but what about a drink at Transport Hotel in 25 minutes?". As in a traditional Internet chat people can leave the conversation and new people can be invited to participate along the way. The conversation continues indefinitely until everyone leaves. 
This design supports interaction maintaining the group and limits the discomfort of waiting by providing an open communication channel to one's friends as well as information about their present location (and hence information about how long they will be). Furthermore, it makes use of the user's history, physical familiarity and social experiences in supporting coordination of where to meet.

\section{Conclusions and Further Work}

In this paper we have presented a field study of small groups socialising in a public place aimed at providing a better understanding of the users' context, situated interactions and the interplay between the two. Based on a grounded analysis of our findings we have presented a conceptual framework of situated social interactions in public. Finally, we have illustrated how this conceptual framework informed the design of a mobile context-aware prototype for supporting sociality by providing a grounded understanding of people's situated social interactions in public places in an abstract form that inspires broad design solutions rather than specifying narrow system requirements.

The research presented in this paper is ongoing. The described paper prototype has been implemented in a functional prototype, which is scheduled for a large-scale field evaluation at Federation Square throughout February 2005. The focus of the evaluation is not as much on the usability of the design but rather on the usefulness of the underlying design ideas discussed above. On the basis of the findings from the evaluation the prototype will be refined and subjected to further evaluations.

\section{Acknowledgements}

This research is supported by the Danish Technical Research Council's talent project "Indexical Interaction Design for Context-aware Mobile Computer Systems" (project reference 26-04-0026). The authors thank Steve Howard and Bharat Dave for input into the design of the field study.

\section{References}

1. Agre, P.: Changing Places - Contexts of Awareness in Computing. Human-Computer Interaction. 16 (2001) 177-192

2. Beyer, H., Holtzblatt, K.: Contextual Design - Defining Customer Centred Systems. Morgan Kaufmann, San Francisco (1998)

3. Blomberg, J., Burrell, M.: An Ethnographic Approach to Design. In: Jacko, J., Sears, A. (eds.): Handbook of Human-Computer Interaction. Lawrence Erlbaum Associates Inc., Mahwah New Jersey (2003) 964-986

4. Bornträger, C., Cheverst, K.: Social and Technical Pitfalls Designing a Tourist Guide System. Proceedings of HCI in Mobile Guides, Udine, Italy (2003)

5. Ciolfi, L.: Understanding Spaces as Places: Extending Interaction Design Paradigms. Cognition Technology and Work 6. 1 (2004) 37-40 
6. CNN.com: Japan's Lonely Hearts Find Each Other with "lovegety". CNN.com, 7 June 1998, http://www.cnn.com/WORLD/asiapcf/9806/07/fringe/japan.lovegety/ (1998) (accessed 16 January 2005)

7. Dourish, P.: Seeking a Foundation for Context-Aware Computing. Human-Computer Interaction. 16 (2001) 229-241

8. Dourish, P.: What We Talk About When We Talk About Context. Personal and Ubiquitous Computing. 8(1) (2004) 19-30

9. Fithian, R., Iachello, G., Moghazy, J., Pousman, Z., Stasko, J.: The Design and Evaluation of a Mobile Location-Aware Handheld Event Planner. Proceedings of Mobile HCI 2003, Udine, Italy, LNCS. Springer-Verlag, Berlin (2003) 145-160

10. Grinter, R. E., Eldridge, M.: y do tngrs luv 2 txt msg? Proceedings of the Seventh European Conference on Computer-Supported Cooperative Work ECSCW'01, Bonn, Germany. Kluwer Academic Publishers, Dordrecht Netherlands (2001) 219-238

11. Kjeldskov, J.: Just-In-Place Information for Mobile Device Interfaces. Proceedings of Mobile HCI 2002, Pisa, Italy, LNCS. Springer-Verlag, Berlin (2002) 271-275

12. Kjeldskov, J., Paay, J.: Just-for-Us: A Context-Aware Information System Facilitating Sociality. (2005) forthcoming

13. McCullough, M.: On Typologies of Situated Interactions. Human-Computer Interaction. 16 (2001) 337-347

14. McCullough, M.: Digital Ground - Architecture, Pervasive Computing, and Environmental Knowing. The MIT Press Cambridge, Massachusetts London (2004)

15. Neuman, W.: Social Research Methods: Qualitative and Quantitative Approaches. Allyn and Bacon, Boston (1994)

16. Paay, J., Kjeldskov. J.: Understanding and Modelling the Built Environment for Mobile Guide Interface Design. Behaviour and Information Technology. 24 (2005) 21-35

17. Persson, P., Espinoza, F., Sandin, A., Coster, R.: GeoNotes: A Location-based Information System for Public Spaces. Proceedings of Mobile HCI 2002, Pisa, Italy, LNCS. Springer-Verlag, Berlin (2002) 151-173

18. Rheingold H.: Smart Mobs. The Next Social Revolution. Perseus Publishing, Cambridge (2003)

19. Schmidt, A.: Implicit Human Computer Interaction Through Context. Personal Technologies. 4(2) (2000) 191-199

20. Snyder C.: Paper Prototyping. The Fast and Easy Way to Design and Refine User Interfaces. Morgan Kaufmann, Amsterdam (2003)

21. Strauss, A., Corbin, J.: Basics of Qualitative Research. Sage Publications, Newbury Park, California (1990)

22. Tamminen, S., Oulasvirta, A., Toiskallio, K., Kankainen, A.: Understanding mobile contexts. Proceedings of Mobile HCI 2003, Udine, Italy, LNCS. Springer-Verlag, Berlin (2003) 17-31 